\title{
Long term survival of patients with gastric cancer treated with adjuvant radio-chemotherapy: proposal of a prognostic index with implication for treatment modification
}

\author{
Junliang Liu* and Shahida Ahmed \\ Department of Radiation Oncology, Cancer Care Manitoba, University of Manitoba, Winnipeg, Manitoba, Canada
}

\begin{abstract}
Purpose/Objective (s): Definitive surgery followed by adjuvant radio chemotherapy has been the standard of care for patients with gastric cancer since the publication of INT0116 study in 2001. This study is to analyze the outcomes of patients with gastric cancer treated with adjuvant radio chemotherapy in Manitoba.

Materials/Methods: After definitive surgical resection, patients with Stage IB to IVM0 gastric cancer were treated with fluorouracil (5-FU) and leucovorin. Radiotherapy with concomitant 5-FU was initiated in the second month of the treatment. Radiotherapy, $4500 \mathrm{cGy}$ in 25 fractions, five days per week, was delivered to the tumor bed, the regional nodes, anastomosis, and the residual stomach. All patients were regularly followed. All the sites of recurrent disease were verified by image or biopsy.

Results: Between 2002 to 2013, 81 patients, male 61, female 20, aged 38 to 79 years old, who finished a full course of adjuvant radio chemotherapy were identified. The median post-radiotherapy follow up was 57 months (10-196 months). Forty-eight (59.3\%) patients survived $\geq 3$ years. Fifteen patients (18.5\%) survived $\geq 5$ years, and nineteen patients $(23.5 \%)$ survived $\geq 10$ years. Eighteen out of $81(22.2 \%)$ patients are still alive with a median survival of 142 months (57-196 months). The tumor and nodal staging, margin status, and lymph vascular invasion are all related to prognosis, but nodal status and lymph vascular invasion in particular were significantly related to prognosis for survival. A prognostic index based on pathologic features has been established to correlate with patient survival with implication for treatment selection.
\end{abstract}

Conclusion: Long- term survival of patients with gastric cancer who received adjuvant radio chemotherapy is possible for a significant portion of patients. A prognostic index has been established to be used for possible risk-based treatment modification.

\section{Introduction}

Gastric cancer is still one of the leading causes of death for patients with malignancies in the world [1,2]. Although the incidence of gastric cancer has been declining steadily for the recent decades, its mortality still ranks very high in the United States [3]. Surgery alone has a dismal 5 -year survival for locally advanced tumor with $34-70 \%$ for patients with Stage I and II disease, and 7-20\% for patients with Stage II and IV disease [4-8]. The multi-institute randomized trial by SWOGDirected Intergroup Study 0116 (INT0116) in 2001 demonstrated that adjuvant radiotherapy plus adjuvant chemotherapy significantly improved the overall as well as disease free survival for patients with local advance gastric cancer [9]. Although the MAGIC study showed that perioperative chemotherapy in addition to definitive surgery achieved similar results [10], adjuvant radio chemotherapy is still the common standard of care in the United States. Patients with locally advanced gastric cancer are usually with different risk factors which affect the ultimate prognosis after the treatment [11,12]. There has been lack of reports about the long- term survival and the associated prognostic factors for patients with gastric cancer treated with adjuvant chemoradiotherapy. This study aims at analyzing the treatment outcomes of patients treated for locally advanced gastric cancer at Cancer Care Manitoba, Canada.

\section{Material and methods}

Surgery: The definitive surgery included total, sub-total, or partial gastrectomy with lymph node dissection as per the discretion of the relevant surgeon for the patients.

The patients were referred to Cancer Care Manitoba after definitive surgery for adjuvant management. Patients were assessed by both medical and radiation oncologists for suitability of adjuvant treatment. Patients with Stage 1B to IV M0 were treated as per INT0116 protocol (9) with modification.

Chemotherapy: Before radiotherapy, patients received the modified De Gramont regimen Leucovorin (LV) $400 \mathrm{mg} / \mathrm{m}^{2} \mathrm{IV}$ and 5 -FU IV bolus $400 \mathrm{mg} / \mathrm{m}^{2}$ on day 1 followed by 5 -FU $2400 \mathrm{mg} / \mathrm{m}^{2}$

*Correspondence to: Junliang Liu, Department of Radiation Oncology, Cancer Care Manitoba, Winnipeg, MB, Canada, R3E 0V9, Tel: +1-204-787-1927; Fax: +1-204-786-0194; E-mail: jliu2@cancercare.mb.ca

Key words: gastric/stomach cancer, adjuvant radio chemotherapy, prognostic factors, and prognostic index

Received: March 23, 2018; Accepted: April 13, 2018; Published: April 16, 2018 
Liu J (2018) Long term survival of patients with gastric cancer treated with adjuvant radio-chemotherapy: proposal of a prognostic index with implication for treatment modification

infusion over 46 hours every 14 days for 2 cycles. During radiotherapy, patients received 5 -FU infusion $200 \mathrm{mg} / \mathrm{m}^{2} /$ day. After radiotherapy, patients were given additional 4 cycles of the modified De Gramont regimen.

Radiotherapy: Patients were CT- simulated in supine position with immobilization. The target volumes were defined as per INT 0116 protocol (9) and included the residual stomach, tumor bed, anastomosis and the regional lymph nodes. Most radiotherapy plans were 3-dimmentional conformal radiotherapy (3D-CRT). Photon beams from linear accelerator with energy of $6 / 10$ or $6 / 23 \mathrm{MV}$ were used for radiotherapy planning. The planning target (PTV) volume for each patient was generated from the clinical target volume (CTV) plus $1 \mathrm{~cm}$ margins. Radiotherapy dose of $45 \mathrm{~Gy}$ in 25 fractions was delivered at daily fraction of $1.8 \mathrm{~Gy}, 5$ days a week over 5 weeks.

Patient follow up: All patients were followed up regularly as per our standard including regular clinical assessment, blood work, and images.

\section{Statistical analysis}

The influences of prognostic factors on overall survival were studied by univariate analysis and multivariate analysis using Cox proportional hazards model. The differences between long- and short- term survival groups were analyzed by $\mathrm{X}^{2}$ test.

\section{Results}

Between 2002 to 2013, patients finished the full course of adjuvant radio chemotherapy were consecutively selected for this study. A total of 81 patients, male 61 , female 20 , aged 38 to 79 years old, were identified. The median overall survival for male patients was 57 months (10-196 months), and for female was 41 months (10-180 months). Forty-eight patients $(59.3 \%)$ survived $\geq 3$ years (Tables $1-3$ ). Nineteen patients (23.5\%) survived $\geq 10$ years (Table 1 ), fifteen patients (18.5\%) survived 5 or more years (Table 2), and fourteen patients (17.3\%) survived 3 $\geq$ years but less than 5 years (Table 3 ). While univariate analysis showed that tumor stage, nodal stage, and margin status are significantly associated with the prognosis (Table 4), the multivariate analysis identified the nodal status was the only prognostic factor which was significant (Table 5). To correlate with the survival, a prognostic index (PI) system has been created. Table 6 demonstrates that the PI is heavily weighted on the advanced tumor stage, nodal stage, and lymph vascular invasion (LVI). A patient with a T4 tumor (3 points), N2-3 (4 points) with positive LVI (4 points) and positive surgical margins (2 points) would have a maximal PI of 13 points (Table 6). The patients are further divided into low risk ( $\mathrm{PI}=1-4)$, intermediate risk $(\mathrm{PI}=5-8)$, and high risk $(\mathrm{PI}=9-13)$ group by using the PI scores. The median survival for patients in the low risk group is almost 6 times' longer than those in the high-risk group and more than double of those in the intermediate risk group (Table 7). When comparing the group of long-term survivors with the group of short-term survivors, the nodal stage and LVI were significantly different (Table 8 ). The recurrences were categorized as infield (occurred in the radiotherapy fields), regional, and distant. Forty one out of 81 patients $(50.6 \%)$ relapsed with the patterns at first failure summarized in Table 9.

\section{Discussion}

This study is focused on the analysis of the overall survival (OSV) of patients with gastric cancer treated with adjuvant radio chemotherapy. In general, the literature considers 3 or more year's OSV of patients with gastric cancer as long-term [11]. Almost $60 \%$ of our patients who finished a full course of adjuvant radio chemotherapy were long-term survivors and this is in line with or better than the literature reports for the similar studies $[13,14]$. There is no doubt that adjuvant radio chemotherapy significantly increases OSV for patients whose stomach cancers were surgically resected with curative intention.

Although perioperative chemotherapy [10] or adjuvant chemotherapy [15] alone (popular in Asia) have been shown to increase survival in addition to definitive surgery, adjuvant radiochemotherapy is still the common standard of care in the United States. Adjuvant radiochemotherapy is sometimes a more practical or better solution for patients with certain demography such as those who are operated by surgeons from community hospitals.

One of the advantages of adjuvant treatment for patients with stomach cancer is that the pathologic features including the tumor and nodal staging as well as LVI can be fully assessed and the treatment can

Table 1. The characteristics of patients who survived 10 or more years after adjuvant radiochemotherapy ( $\mathrm{n}=19)$.

\begin{tabular}{|c|c|c|c|c|c|c|c|c|c|c|}
\hline Age & Gender & Tumor location & Tumor Stage & Nodal stage & Histology & Surgery & Margins & LVI\# & OSV $\infty$ (months) & PI $\times$ \\
\hline 62 & $\mathrm{~m}$ & antrum & 3 & 0 & 3 & Subtotal & negative & negative & $196 a^{*}$ & 2 \\
\hline 49 & $\mathrm{~m}$ & GE junction & 3 & 1 & 2 & total & negative & negative & $196 \mathrm{a}$ & 4 \\
\hline 47 & $\mathrm{~m}$ & antrum & 2 & 1 & 2 & subtotal & negative & negative & $177 \mathrm{a}$ & 3 \\
\hline 58 & $\mathrm{~m}$ & GE junction & 2 & 1 & 1 & total & Close & negative & $141 \mathrm{~d} ¥$ & 4 \\
\hline 56 & $\mathrm{~m}$ & antrum & 3 & 0 & 2 & subtotal & Positive & negative & $180 \mathrm{a}$ & 4 \\
\hline 65 & $\mathrm{f}$ & GE junction & 3 & 1 & 3 & total & negative & negative & $180 \mathrm{a}$ & 4 \\
\hline 61 & $\mathrm{~m}$ & antrum & 2 & 1 & 3 & subtotal & close & negative & $172 \mathrm{a}$ & 4 \\
\hline 54 & $\mathrm{~m}$ & proximal & 3 & 2 & 2 & total & negative & negative & $153 \mathrm{a}$ & 6 \\
\hline 66 & $\mathrm{~m}$ & Lesser curvature & 3 & 0 & 2 & subtotal & Close & negative & $161 \mathrm{a}$ & 3 \\
\hline 41 & $\mathrm{f}$ & Lesser curvature & 2 & 1 & 3 & partial & negative & positive & $142 \mathrm{a}$ & 7 \\
\hline 78 & $\mathrm{~m}$ & GE junction & 3 & 0 & 1 & total & Positive & negative & $125 \mathrm{a}$ & 4 \\
\hline 43 & $\mathrm{f}$ & antrum & 3 & 2 & 3 & subtotal & Positive & negative & $131 \mathrm{a}$ & 8 \\
\hline 67 & $\mathrm{f}$ & antrum & 4 & 1 & 3 & subtotal & negative & negative & $175 d$ & 5 \\
\hline 73 & $\mathrm{~m}$ & antrum & 3 & 3 & 3 & subtotal & negative & negative & $174 \mathrm{~d}$ & 6 \\
\hline 57 & $\mathrm{f}$ & antrum & 3 & 1 & 3 & subtotal & positive & negative & $133 \mathrm{~d}$ & 6 \\
\hline 58 & $\mathrm{f}$ & antrum & 2 & 0 & 3 & subtotal & negative & negative & $150 \mathrm{~d}$ & 1 \\
\hline 59 & $\mathrm{~m}$ & antrum & 2 & 0 & 3 & partial & negative & negative & $137 \mathrm{~d}$ & 1 \\
\hline 64 & $\mathrm{~m}$ & GE & 3 & 1 & 3 & total & Close & negative & $123 \mathrm{~d}$ & 5 \\
\hline 59 & $\mathrm{~m}$ & antrum & 2 & 0 & 3 & subtotal & negative & negative & $168 \mathrm{~d}$ & 1 \\
\hline
\end{tabular}

\# LVI=lymphovascular invasion; $\infty$ OSV= overall survival; $\times \mathrm{PI}=$ prognostic Index; *a=Alive; $¥ \mathrm{~d}=\mathrm{die}$ 
Liu J (2018) Long term survival of patients with gastric cancer treated with adjuvant radio-chemotherapy: proposal of a prognostic index with implication for treatment modification

Table 2. characteristics of patients who survived $\geq 5$ but less than 10 years after radiochemotherapy ( $n=15)$

\begin{tabular}{|c|c|c|c|c|c|c|c|c|c|c|}
\hline Age & Gender & Tumor location & $\begin{array}{c}\text { Tumor } \\
\text { stage }\end{array}$ & Nodal stage & Histology & Surgery & Margins & LVI\# & $\begin{array}{c}\text { OSV } \infty \\
\text { (months) }\end{array}$ & PIX \\
\hline 75 & $\mathrm{~m}$ & pylorus & 3 & $\mathrm{Nx}$ & $\mathrm{G} 2$ & Sub total & positive & negative & $114 \mathrm{~d} ¥$ & 5 \\
\hline 75 & $\mathrm{~m}$ & incisura & 3 & 0 & G2 & subtotal & negative & negative & $81 \mathrm{~d}$ & 2 \\
\hline 69 & $\mathrm{~m}$ & GE junction & 4 & $\mathrm{nx}$ & G3 & total & positive & negative & $100 \mathrm{~d}$ & 6 \\
\hline 69 & $\mathrm{~m}$ & GE & 2 & 2 & G3 & total & negative & negative & $112 \mathrm{~d}$ & 5 \\
\hline 72 & $\mathrm{~m}$ & Lesser curvature & 3 & 1 & G3 & subtotal & positive & negative & $108 \mathrm{~d}$ & 6 \\
\hline 60 & $\mathrm{~m}$ & pylorus & 2 & 1 & $\mathrm{G} 2$ & subtotal & negative & negative & $89 \mathrm{~d}$ & 3 \\
\hline 76 & $\mathrm{~m}$ & antrum & 2 & 1 & G3 & subtotal & negative & negative & $103 d$ & 3 \\
\hline 61 & $\mathrm{~m}$ & GE & 3 & 1 & G3 & total & Close & positive & $111 a^{*}$ & 9 \\
\hline 62 & $\mathrm{~m}$ & antrum & 2 & 1 & G2 & partial & negative & negative & $112 \mathrm{a}$ & 3 \\
\hline 68 & $\mathrm{~m}$ & body & 2 & 1 & G3 & subtotal & close & positive & $112 \mathrm{a}$ & 9 \\
\hline 65 & $\mathrm{~m}$ & GE & 3 & 1 & G2 & total & negative & negative & $68 \mathrm{~d}$ & 4 \\
\hline 62 & $\mathrm{~m}$ & antrum & 2 & 2 & G3 & subtotal & negative & negative & $84 \mathrm{~d}$ & 5 \\
\hline 69 & $\mathrm{~m}$ & antrum & 2 & 0 & G2 & subtotal & negative & negative & $105 \mathrm{a}$ & 1 \\
\hline 59 & $\mathrm{~m}$ & antrum & 3 & 2 & G3 & subtotal & negative & negative & $87 \mathrm{a}$ & 6 \\
\hline 79 & $\mathrm{~m}$ & pylorus & 3 & 1 & G3 & subtotal & negative & positive & $75 \mathrm{a}$ & 8 \\
\hline
\end{tabular}

\# LVI=lymph vascular invasion; $\infty$ OSV= overall survival; $\times$ PI=prognostic Index; *a=Alive; $¥ \mathrm{~d}=\mathrm{die}$.

Table 3. Characteristics of Patients survived 3 or more but less than 5 years after adjuvant radiochemotherapy $(n=14)$.

\begin{tabular}{|c|c|c|c|c|c|c|c|c|c|c|}
\hline Age & Gender & Tumor location & Tumor stage & Nodal stage & Histology & Surgery & Margins & LVI\# & Osv $\infty$ (months) & PI $\times$ \\
\hline 40 & $\mathrm{f}$ & pylorus & 3 & 1 & 3 & partial & negative & negative & $57 \mathrm{~d} ¥$ & 4 \\
\hline 71 & $\mathrm{~m}$ & Gastric remnant & 3 & 1 & 3 & total & Close & positive & $55 \mathrm{~d}$ & 9 \\
\hline 64 & $f$ & antrum & 3 & 2 & 2 & partial & positive & negative & $50 \mathrm{~d}$ & 8 \\
\hline 61 & $\mathrm{~m}$ & antrum & 3 & 1 & 3 & subtotal & negative & negative & $57 \mathrm{~d}$ & 4 \\
\hline 63 & $f$ & fundus & 3 & 1 & 2 & partial & negative & negative & $50 \mathrm{~d}$ & 4 \\
\hline 70 & $\mathrm{~m}$ & antrum & 2 & 1 & 1 & subtotal & negative & positive & $36 \mathrm{~d}$ & 7 \\
\hline 56 & $\mathrm{~m}$ & GE & 3 & 1 & 2 & Total & Close & positive & $38 \mathrm{~d}$ & 9 \\
\hline 58 & $f$ & antrum & 3 & 1 & 3 & subtotal & negative & negative & $42 d$ & 4 \\
\hline 49 & $\mathrm{~m}$ & GE & 3 & 1 & 2 & Total & positive & - & $58 \mathrm{~d}$ & 6 \\
\hline 59 & $\mathrm{~m}$ & GE & 4 & 2 & 3 & Total & positive & yes & $46 \mathrm{~d}$ & 13 \\
\hline 59 & $f$ & antrum & 3 & 2 & 3 & subtotal & Close & negative & $40 \mathrm{~d}$ & 7 \\
\hline 43 & $\mathrm{~m}$ & Great curvature & 3 & 0 & 3 & Total & negative & negative & $36 \mathrm{~d}$ & 2 \\
\hline 50 & $\mathrm{~m}$ & $\begin{array}{c}\text { Distal+ } \\
\text { duodenum }\end{array}$ & 4 & 1 & 2 & partial & positive & negative & $43 \mathrm{~d}$ & 7 \\
\hline 72 & $\mathrm{~m}$ & antrum & 4 & 1 & 3 & partial & negative & negative & $57 a^{*}$ & 5 \\
\hline
\end{tabular}

\# LVI=lymph vascular invasion; $\infty$ OSV= overall survival; $\times$ PI=prognostic Index; *a=Alive; $¥ \mathrm{~d}=\mathrm{di}$.

Table 4. Univariate predictor analysis of maximum likelihood estimates.

\begin{tabular}{|c|c|c|c|}
\hline Variable & Hazard Ratio & 95\% CI \\
\hline Male vs female & 0.69 & $0.39-1.21$ \\
\hline T stage (T3-4 vs T1-2) & 3.66 & $1.46-9.20$ \\
\hline N stage (N2-3 vs N0-1) & 1.95 & $1.14-3.35$ \\
\hline Nodes removed (13+ vs fewer or none) & 0.84 & $0.48-1.48$ \\
\hline Histology (G3 vs G1-2) & 0.89 & $0.48-1.66$ \\
\hline Margin & 1.92 & 0.058 \\
\hline
\end{tabular}

Table 5. Multivariate predictor analysis of maximum likelihood estimates.

\begin{tabular}{|c|c|c|c|}
\hline Variable & Hazard ratio & 95\%CI \\
\hline Male vs female & 1.19 & $0.54-2.65$ \\
\hline T stage (T3-4 vs T1-2) & 2.54 & $0.78-8.21$ \\
\hline N stage (N2-3 vs N0-1) & 3.32 & $1.52-7.24$ \\
\hline Nodes removed (13+ vs fewer or none) & 0.64 & $0.3-1.38$ \\
\hline Histology (G3 vs G1-2) & 0.57 & 0.2630 \\
\hline Margin & 1.02 & $0.24-1.36$ \\
\hline
\end{tabular}

Table 6. Prognostic index (PI) scores based on surgical pathological features.

\begin{tabular}{|c|c|c|c|c|c|c|c|c|c|c|c|c|c|}
\hline & \multicolumn{3}{|c|}{ T Stage } & \multicolumn{4}{|c|}{ N Stage } & \multicolumn{2}{|c|}{ LVI } & \multicolumn{3}{|c|}{ Surgical Margins } & \multirow{2}{*}{$\begin{array}{c}\text { Maximal } \\
\text { scores }\end{array}$} \\
\hline & $1-2$ & 3 & 4 & 0 & $\mathrm{x}$ & 1 & $2-3$ & negative & positive & negative & close & positive & \\
\hline PI & 1 & 2 & 3 & 0 & 1 & 2 & 4 & 0 & 4 & 0 & 1 & 2 & 13 \\
\hline
\end{tabular}


Liu J (2018) Long term survival of patients with gastric cancer treated with adjuvant radio-chemotherapy: proposal of a prognostic index with implication for treatment modification

Table7. Survival according to risk based on PI.

\begin{tabular}{|l|l|}
\hline PI Group & Median Overall Survival (months) \\
\hline Low risk (PI=1-4, $\mathbf{n = 2 6})$ & $112(10-196)$ \\
\hline Intermediate risk (PI=5-8, $\mathbf{n = 3 5})$ & $50(10-175)$ \\
\hline High risk (PI=9-13, $\mathbf{n = 2 0})$ & $20(11-112)$ \\
\hline
\end{tabular}

Table 8. The characteristic differences between long- and short- term survival patients.

\begin{tabular}{|c|c|c|c|c|c|c|}
\hline \multirow{2}{*}{ OSV } & \multicolumn{2}{|c|}{ Tumor stage } & \multicolumn{2}{|c|}{ Nodal stage } & \multirow{2}{*}{$\begin{array}{c}\text { Close/ } \\
\text { positive } \\
\text { margins }\end{array}$} & \multirow{2}{*}{ LVI } \\
\hline & T1-2 & T3-4 & N0-1 & N2-3 & & \\
\hline$\geq 3$ years & $\begin{array}{c}15 / 48 \\
(31.25 \%)\end{array}$ & $\begin{array}{c}33 / 48 \\
(68.75 \%)\end{array}$ & $\begin{array}{c}37 / 48 \\
(77.08 \%)\end{array}$ & $\begin{array}{c}11 / 48 \\
(22.92 \%)\end{array}$ & $\begin{array}{c}20 / 48 \\
(41.67 \%)\end{array}$ & $\begin{array}{c}8 / 48 \\
(16.67 \%)\end{array}$ \\
\hline$<3$ years & $\begin{array}{c}5 / 33 \\
(15.15 \%)\end{array}$ & $\begin{array}{c}28 / 33 \\
(84.85 \%)\end{array}$ & $\begin{array}{c}13 / 33 \\
(39.39 \%)\end{array}$ & $\begin{array}{c}20 / 33 \\
(60.61 \%)\end{array}$ & $\begin{array}{c}21 / 33 \\
(63.64 \%)\end{array}$ & $\begin{array}{c}15 / 33 \\
(45.45 \%)\end{array}$ \\
\hline P value & $>0.05$ & $>0.05$ & $<0.005$ & $<0.005$ & $>0.05$ & $<0.005$ \\
\hline
\end{tabular}

Table 9. Patterns at first failure*.

\begin{tabular}{|c|c|c|c|c|c|c|c|}
\hline site & In-field & $\begin{array}{c}\text { In-field } \\
\text { +Regional }\end{array}$ & $\begin{array}{c}\text { In-field } \\
\text { +distant }\end{array}$ & regional & $\begin{array}{c}\text { Regional } \\
\text { +distant }\end{array}$ & distant & total \\
\hline $\begin{array}{c}\text { Number } \\
\text { of } \\
\text { patients }\end{array}$ & 3 & 2 & 1 & 19 & 3 & 13 & 41 \\
\hline
\end{tabular}

*In-fields: tumor relapsed in the radiotherapy target volumes: surgical anastomosis, residual stomach, gastric bed, and the targeted nodal area; Regional: tumor recurred in the peritoneal cavity including the liver, pelvic organs, peritoneum, and the non-targeted intraabdominal lymph nodes; Distant: relapse outside the peritoneal cavity.

be tailored accordingly. We identified the nodal stage and lymph vascular invasion as significant risk factors which are associated with survival. A prognostic index score system has been established to further categorize this patient population. By using this prognostic index, the patients can be further stratified into different risk groups. The patients from the high-risk group of this report had very poor prognosis after adjuvant radiochemotherapy. It is conceivable that a different treatment strategy should be explored for these patients. It appears that patients from the high-risk category relapsed mostly beyond the radiotherapy target volumes with either regional of distant failure. Those patients might benefit from a course of vigorous effective chemotherapy regimens such as FLOT which includes docetaxel, oxaliplatin 5-FU and LV for 3-4 cycles followed by the standard radiochemotherapy and additional 3-4 cycles of FLOT after radiotherapy. FLOT has been showed superior to other chemotherapy combination [16].

A meta-analysis of the randomized studies showed no overall survival benefit with D2 dissection which was associated with greater mortality and or morbidity [17]. Studies reported significant local regional failure with D2 surgery alone [18] and the improved survival with adjuvant radio chemotherapy is independent of nodal clearance [19]. Neither the number of the removed lymph nodes nor the extent of lymph node dissection is associated with the survival of our patients (data not shown).

The treatment related toxicities reported by the INT0116 study were significant. That study used two-dimensional radiotherapy with parallel opposed fields and the standard bolus 5FU/LV regimen which is no longer recommended. Our patients were treated with 3D-CRT and in fusional 5-FU with much less side effects. The observed side effects for our patients are stomatitis, nausea, fatigue, occasional mild diarrhea, and foot hand syndrome (data not shown).

The patterns of failure are important to guide the treatment strategy. Except a few in radiotherapy field failure, patients either failed regional including the liver and peritoneum or failed with metastases at distant as showed in the current study and the previous report [20]. Clearly, effective systemic regimen including chemotherapy or target therapy drugs should be vigorously explored. The failures are mostly predictable by using the prognostic index established by this study. The patients fall into the high-risk category should receive a more effective systemic treatment other than the traditional 5-FU/LV before and after adjuvant radiotherapy.

In conclusion, long-term survival is possible for patients with gastric cancer whose tumors were surgical resected with curative intention. A prognostic index for patients with gastric cancer has been generated to possibly guide the selection of more effective systemic adjuvant treatment in the future.

\section{Conflict of interest}

The author report that no potential conflicts of interest exist.

\section{References}

1. Ferlay J, Soerjomataram I, Eryik M, Dikshit R, Eser S, et al. (2014) Globocan 2012 Cancer Incidence and mortality worldwide: IARC cancer Base No. 11. Lyon, France: International Agency for Research on Cancer.

2. Jemal A, Center MM, DeSantis C, Ward EM (2010) Global patterns of cancer incidence and mortality rates and trends. Cancer Epidemiol Biomarkers Prev 19: 18931907. [Crossref]

3. Siegel RL, Miller KD, Jemal A (2018) Cancer statistics, 2018. CA Cancer J Clin 68 7-30. [Crossref]

4. Quero L, Bouchbika Z, Kouto H, Baruch-Hennequin V, Gornet JM, et al. (2012) Postoperative chemotherapy followed by conformal concomitant chemoradiotherapy in high-risk gastric cancer. Int J Radiat Oncol Biol Phys 83: 574-580. [Crossref]

5. Gunderson LL (2002) Gastric cancer patterns of relapse after surgical resection. Semin Radiat Oncol 12: 150-161. [Crossref]

6. Gunderson LL, Sosin H (1982) Adenocarcinoma of the stomach: Areas of failure in a re-operation series (second or symptomatic look) clinicopathologic correlation and implications for adjuvant therapy. Int J Radiat Oncol Biol Phys 8: 1-11. [Crossref]

7. Mcneer G, Vandenberg H Jr, Donn Fy, Bowden L (1951) A critical evaluation of subtotal gastrectomy for the cure of cancer of the stomach. Ann Surg 134: 2-7. [Crossref]

8. Hundahl SA, Phillips JL, Menck HR (2000) The National Cancer Data Base Report on poor survival of U.S. gastric carcinoma patients treated with gastrectomy: Fifth Edition American Joint Committee on Cancer staging, proximal disease, and the "different disease" hypothesis. Cancer 88: 921-932. [Crossref]

9. Macdonald JS, Smalley SR, Benedetti J, Hundahl SA, Estes NC, et al. (2001) Chemoradiotherapy after surgery compared with surgery alone for adenocarcinoma of the stomach or gastroesophageal junction. N Engl J Med 345: 725-730. [Crossref]

10. Cunningham D, Allum WH, Stenning SP, Thompson JN, Van de Velde CJ, et al. (2006) Perioperative chemotherapy versus surgery alone for resectable gastroesophageal cancer. $N$ Engl J Med 355: 11-20. [Crossref]

11. Kunz PL, Gubens M, Fisher GA, Ford JM, Lichtensztajn DY, et al. (2012) Long-term survivors of gastric cancer: a California population-based study. J Clin Oncol 30: 35073515. [Crossref]

12. SunY, Yang L, Wang C, Zhao D, Cai J, et al. (2017) Prognostic factors associated with locally advanced gastric cancer patients treated with neoadjuvant chemotherapy followed by surgical resection. Oncotarget 8: 75186-75194. [Crossref]

13. Smalley SR, Benedetti JK, Haller DG, Hundahl SA, Estes NC, et al. (2012) Updated analysis of SWOG-Directed Intergroup Study 0116: A phase II trial of adjuvant radiochemotherapy versus observation after curative gastric cancer resection. $J$ Clin Oncol 30: 2327-2333. [Crossref]

14. Gondim GRM, FogaroliRC, Pellizzon AC (2015) Prognostic factors of recurrence pattern, survival and toxicity in patients with gastric cancer treated according to protocol INT-0116 with 3D-conformal radiotherapy. SOJ Surgery 2: 1-6.

15. Grau JJ, Martín M, Fuster J, Pera M, García-Valdecasas JC, et al. (2003) Impact of adjuvant chemotherapy in the long-term outcome of patients with resected gastric cancer. J Surg Oncol 82: 234-240. [Crossref]

16. Al Batran S-E, Homann N, Schmalenberg H (2017) Perioperative chemotherapy with docetaxel, oxaliplatin, and fluorouracil/leucovorin (FLOT) versus epirubicin, cisplatin, and fluorouracil or capecitabine $(\mathrm{ECF} / \mathrm{ECX})$ for resectable gastric or gastroesophageal junction (GEJ) adenocarcinoma (FLOT4-AIO): A multicenter, randomized phase 3 trial (abstract). J Clin Oncol 35. 
Liu J (2018) Long term survival of patients with gastric cancer treated with adjuvant radio-chemotherapy: proposal of a prognostic index with implication for treatment modification

17. McCulloch P, Nita ME, Kazil H, Gama-Rodrigues J (2003) Extended versus limited lymph nodes dissection technique for Aden carcinoma of the stomach. Cochrane Database SYS rev 18: CD001964. [Crossref]

18. Hartgrink HH, van de Velde CJ, Putter h, Bonenkamp JJ, Klein Kranenbarg E, et al. (2004) Extended lymph node dissection for gastric cancer: Who may benefit? Final results of the randomized Dutch Gastric Cancer Group trial. J Clin oncol 22: 20692077. [Crossref]
19. Coburn NG, Govindarajan A, Law CH, Guller U, Kiss A, et al. (2008) Stage-specific effect of adjuvant therapy following gastric cancer resection: A population-based analysis of 4041 patients. Ann Surg Oncol 15: 500-507. [Crossref]

20. Liu J, Mou B, Liu E (2011) Patterns of failure after definitive surgery followed by adjuvant chemoradiotherapy in patients with stomach cancer Int $J$ Radiation Oncol Bio Phys 81: S326.

Copyright: (C2018 Liu J. This is an open-access article distributed under the terms of the Creative Commons Attribution License, which permits unrestricted use, distribution, and reproduction in any medium, provided the original author and source are credited. 